\title{
THE CONTRACTIBILITY OF CERTAIN TEICHMÜLLER SPACES ${ }^{1}$
}

\author{
BY CLIFFORD J. EARLE
}

Communicated by Maurice Heins, January 3, 1967

1. Introduction. From the work of Fenchel and Nielsen [7] or from recent work of Keen [8], it is known that for every finitely generated Fuchsian group $\Gamma$ of the first kind the Teichmüller space $T(\Gamma)$ is contractible. We extend this result here, proving that the Teichmüller space of every nonelementary finitely generated Fuchsian group is contractible. We give only an indication of the proof. The complete proof, which is rather technical, will appear later.

2. Definitions. Let $U=\{z \in C: \operatorname{Im} z>0\}$ be the upper half plane. Let $\Gamma$ be a finitely generated group of Möbius transformations which map $U$ onto itself. The limit set $L(\Gamma)$ consists of all points in the extended plane which are limit points of some orbit $\Gamma z, z \in C$. We assume that $\Gamma$ is Fuchsian, meaning that $L(\Gamma)$ is a subset of the extended real axis, and nonelementary, meaning that $L(\Gamma)$ contains at least three points. We shall in fact require that $0,1, \infty \in L(\Gamma)$. This involves no loss of generality, for if $\Gamma$ is any nonelementary Fuchsian group, there is a Möbius transformation $A: U \rightarrow U$ such that the Fuchsian group $A \circ \Gamma \circ A^{-1}$ meets our requirement, and the Teichmüller spaces $T(\Gamma)$ and $T\left(A \circ \Gamma \circ A^{-1}\right)$ are homeomorphic [1, Theorem 8].

Let $L^{\infty}(\Gamma)$ be the Banach subspace of $L^{\infty}(U, C)$ consisting of the functions $\mu(z)$ which satisfy

$$
\mu(A z) A^{\prime}(z)^{*} / A^{\prime}(z)=\mu(z) \quad \text { for all } A \text { in } \Gamma .
$$

The open unit ball in $L^{\infty}(\Gamma)$, denoted by $M(\Gamma)$, is called the set of Beltrami differentials. Each Beltrami differential $\mu$ determines a quasiconformal map $w_{\mu}$ of $U$ onto itself which leaves the points $0,1, \infty$ fixed and satisfies in $U$ the Beltrami equation $w_{z}=\mu w_{z}$.

We say that $\mu$ and $\nu$ in $M(\Gamma)$ are strongly equivalent if $w_{\mu}(x)=w_{\nu}(x)$ for all real $x$. The set of strong equivalence classes is the Teichmiiller space $T(\Gamma)$. Let $\Phi: M(\Gamma) \rightarrow T(\Gamma)$ be the map which sends each $\mu$ in $M(\Gamma)$ to its strong equivalence class. We give $T(\Gamma)$ the quotient topology induced by $\Phi$.

If $w_{\mu}(x)=w_{\nu}(x)$ for all $x$ in $L(\Gamma)$ we say that $\mu$ and $\nu$ in $M(\Gamma)$ are

1 This research was partially supported by NSF Grant GP6145. 
weakly equivalent. The set of weak equivalence classes is the reduced Teichmuiller space $T^{\sharp}(\Gamma)$. $\Phi^{\sharp}: M(\Gamma) \rightarrow T^{\sharp}(\Gamma)$ is the map sending each $\mu$ in $M(\Gamma)$ to its weak equivalence class, and $T^{*}(\Gamma)$ is given the quotient topology induced by $\Phi^{\#}$.

REMARK. $\Gamma$ is said to be of the first or second kind according as $R-L(\Gamma)$ is or is not empty. If $\Gamma$ is of the first kind, then $T(\Gamma)=T^{\sharp}(\Gamma)$ and some of the following results, for instance Theorem 2 , become trivial.

3. Some consequences of local triviality. Recall that a map $f: X \rightarrow Y$ is locally trivial if for each $y \in Y$ there exist a neighborhood $N_{y}$ and a homeomorphism $F: f^{-1}(y) \times N_{y} \rightarrow f^{-1}\left(N_{y}\right)$ such that the map $f \circ F: f^{-1}(y) \times N_{y} \rightarrow N_{y}$ is projection on the second factor.

Theorem 1. The maps $\Phi: M(\Gamma) \rightarrow T(\Gamma)$ and $\Phi^{\sharp}: M(\Gamma) \rightarrow T^{\sharp}(\Gamma)$ are locally trivial.

Eells and the present author have proved this property of $\Phi$ in [5] by using the results of their paper [4]. A similar application of the results of [4] shows that $\Phi^{\#}$ is also a locally trivial map. We omit the details.

Now let $M_{0}(\Gamma)=\{\mu \in M(\Gamma): \Phi(\mu)=\Phi(0)\}$ and $M_{0}^{\sharp}(\Gamma)=\{\mu \in M(\Gamma)$ : $\left.\Phi^{\#}(\mu)=\Phi^{\#}(0)\right\}$. As a consequence of Theorem 1 we have the exact homotopy sequences

$$
\begin{aligned}
\ldots & \rightarrow \pi_{i}(M(\Gamma)) \rightarrow \pi_{i}(T(\Gamma)) \rightarrow \pi_{i-1}\left(M_{0}(\Gamma)\right) \rightarrow \pi_{i-1}(M(\Gamma)) \rightarrow \cdots \\
& \rightarrow \pi_{1}(T(\Gamma)) \rightarrow \pi_{0}\left(M_{0}(\Gamma)\right) \rightarrow \pi_{0}(M(\Gamma)),
\end{aligned}
$$

and

$$
\begin{aligned}
\cdots \pi_{i}(M(\Gamma)) & \rightarrow \pi_{i}\left(T^{\sharp}(\Gamma)\right) \rightarrow \pi_{i-1}\left(M_{0}^{\sharp}(\Gamma)\right) \rightarrow \pi_{i-1}(M(\Gamma)) \rightarrow \cdots \\
& \rightarrow \pi_{1}\left(T^{\#}(\Gamma)\right) \rightarrow \pi_{0}\left(M_{0}^{*}(\Gamma)\right) \rightarrow \pi_{0}(M(\Gamma)) .
\end{aligned}
$$

From these, since $M(\Gamma)$ is contractible, we obtain isomorphisms

$$
\begin{aligned}
\pi_{i}(T(\Gamma)) \cong \pi_{i-1}\left(M_{0}(\Gamma)\right), & & i \geqq 1, \\
\pi_{i}\left(T^{\sharp}(\Gamma)\right) \cong \pi_{i-1}\left(M_{0}^{*}(\Gamma)\right), & & i \geqq 1 .
\end{aligned}
$$

CoRollary 1. $\pi_{i}\left(M_{0}^{\sharp}(\Gamma)\right)=0$ for all $i$.

This is a consequence of (3.2), since Fenchel and Nielsen [7] and Keen [8] have proved that under our assumptions on $\Gamma, T^{\sharp}(\Gamma)$ is contractible.

Now put $T_{0}(\Gamma)=\Phi\left(M_{0}^{\sharp}(\Gamma)\right) \subset T(\Gamma)$. Since $\Phi^{-1}\left(T_{0}(\Gamma)\right)=M_{0}^{\sharp}(\Gamma)$, Theorem 1 implies that $\Phi: M_{0}^{\sharp}(\Gamma) \rightarrow T_{0}(\Gamma)$ is a locally trivial map. From this fact we obtain another exact homotopy sequence 


$$
\begin{aligned}
\cdots & \rightarrow \pi_{i}\left(M_{0}^{\#}(\Gamma)\right) \rightarrow \pi_{i}\left(T_{0}(\Gamma)\right) \rightarrow \pi_{i-1}\left(M_{0}(\Gamma)\right) \rightarrow \pi_{i-1}\left(M_{0}^{\sharp}(\Gamma)\right) \rightarrow \cdots \\
& \rightarrow \pi_{1}\left(T_{0}(\Gamma)\right) \rightarrow \pi_{0}\left(M_{0}(\Gamma)\right) \rightarrow \pi_{0}\left(M_{0}^{\#}(\Gamma)\right) .
\end{aligned}
$$

From this exact sequence and Corollary 1 we obtain isomorphisms

$$
\pi_{i}\left(T_{0}(\Gamma)\right) \cong \pi_{i-1}\left(M_{0}(\Gamma)\right), \quad i \geqq 1 .
$$

From (3.1) and (3.3) we obtain

CoRollaRy 2. $\pi_{i}(T(\Gamma)) \cong \pi_{i}\left(T_{0}(\Gamma)\right)$ for all $i$.

If $i=0,(3.1)$ and (3.3) do not apply, but $T(\Gamma)$ and $T_{0}(\Gamma)$ are both connected, being continuous images of the connected sets $M(\Gamma)$ and $M_{0}^{\#}(\Gamma)$.

\section{An inverse of $\Phi$.}

THEOREM 2. There is a continuous map s: $T_{0}(\Gamma) \rightarrow M_{0}^{\#}(\Gamma)$ such that $\Phi \circ s: T_{0}(\Gamma) \rightarrow T_{0}(\Gamma)$ is the identity map.

If $\Gamma$ is a group of the first kind, then $T_{0}(\Gamma)$ is a single point and the theorem is obviously true. We shall sketch the proof of the theorem for a group $\Gamma$ of the second kind.

By the definition of strong equivalence, we may identify $T_{0}(\Gamma)$ with the set of maps $h: R \rightarrow R$ such that $h=w_{\mu} \mid R$ for some $\mu$ in $M_{0}^{\sharp}(\Gamma)$. Our problem is to extend each such $h$ to a quasiconformal map $w_{h}: U \rightarrow U$ in such a way that the dilatation $\left(w_{h}\right)_{z} /\left(w_{h}\right)_{z}$ belongs to $M_{0}^{\sharp}(\Gamma)$ and varies continuously with $h$.

Now $R-L(\Gamma)$ is a union of open intervals $I_{j}$, each of which is mapped on to itself by $h$ (since $h(x)=x$ for all $x$ in $L(\Gamma)$ ). Each interval $I_{j}$ determines a noneuclidean half plane $H_{j}$ bounded by $I_{j}$ and the noneuclidean line in $U$ which terminates at the endpoints of $I_{j}$. Let $H$ be the union of the $H_{j}$. In $U-H$ we put $w_{h}(z)=z$. The remaining problem is to define in each half-plane $H_{j}$ a suitable quasiconformal map of $H_{j}$ on itself having boundary values $h$ on $I_{j}$ and identity on the bounding noneuclidean line. This requires careful use of the methods of Beurling and Ahlfors [2]. We omit the rather lengthy arguments.

Remark. The domain $U-H$ is called by Fenchel and Nielsen the convex figure of $\Gamma$ (see [7]).

5. Contractibility. We are now ready to prove

THEOREM 3. If $\Gamma$ is any finitely generated nonelementary Fuchsian group, the Teichmuiller space $T(\Gamma)$ is contractible. 
PRoof. We note first that Theorem 2 and Corollary 1 of Theorem 1 imply that all homotopy groups of $T_{0}(\Gamma)$ vanish. Indeed, any map $f: S^{n} \rightarrow T_{0}(\Gamma)$ induces a map $s \circ f: S^{n} \rightarrow M_{0}^{\sharp}(\Gamma)$. If $F: S^{n} \times I \rightarrow M_{0}^{\sharp}(\Gamma)$ is a homotopy of $s$ of to a constant map, then $\Phi \circ F: S^{n} \times I \rightarrow T_{0}(\Gamma)$ is a homotopy of $f$ to a constant map.

Now Corollary 2 of Theorem 1 implies that all homotopy groups of $T(\Gamma)$ vanish. But $T(\Gamma)$ is (homeomorphic to) an open set in a Banach space by [1, Theorem 6] or [3]. Hence $T(\Gamma)$ is an absolute neighborhood retract, and by a theorem by J. H. C. Whitehead, the vanishing of all homotopy groups of $T(\Gamma)$ implies that $T(\Gamma)$ is contractible. (See $[6, \S 4]$ and [9].) The theorem is proved.

\section{REFERENCES}

1. L. Bers, Automorphic forms and general Teichmiiller spaces, pp. 109-113, Proc. Conf. Compl. Anal., Minneapolis 1964, Springer, Berlin, 1965.

2. A. Beurling and L. V. Ahlfors, The boundary correspondence under quasiconformal mappings, Acta Math. 96 (1956), 125-142.

3. C. J. Earle, The Teichmiiller space of an arbitrary Fuchsian group, Bull. Amer. Math. Soc. 70 (1964), 699-701.

4. C. J. Earle and J. Eells, Jr., Foliations and fibrations J. Differential Geometry (to appear).

5. - On the differential geometry of Teichmïller spaces J. Analyse Math. (to appear).

6. J. Eells, Jr., A setting for global analysis, Bull. Amer. Math. Soc. 72 (1966), 751-807.

7. W. Fenchel and J. Nielsen, Discontinuous groups of non-Euclidean motions (to appear). 420.

8. L. Keen, Intrinsic moduli on Riemann surfaces, Ann. of Math. 84 (1966), 404-

9. R. Palais, Homotopy theory of infinite dimensional manifolds, Topology 5 (1966), $1-16$.

Cornell University 